\title{
Effects of KAI gene expression on ferroptosis in pancreatic cancer cells
}

\author{
XU LIU, XIAOZHONG GUO, HONGYU LI, JIANG CHEN and HAO HAN
}

\begin{abstract}
Department of Gastroenterology, General Hospital of Northern Theater Command, Shenyang, Liaoning 110840, P.R. China
\end{abstract}
Received March 3, 2020; Accepted November 24, 2020

DOI: $10.3892 / \mathrm{mmr} .2020 .11802$

\begin{abstract}
The specific role and mechanism of ferroptosis in the development of pancreatic cancer (PC) remain to be elucidated. The present study aimed to investigate the effects of the overexpression of the KAI1 gene on the ferroptosis of the human PC cell line MIA PaCa-2. MIA PaCa-2 cells infected with pCMV-KAI1 and selected by G418 and KAI1 protein were analyzed by western blotting. The MIA PaCa-2 cells with a stable expression of the KAI1 gene were termed MIA PaCa-2-KAI1. The proliferative capacities of MIA PaCa-2 and MIA PaCa-2-KAI1 cells were detected using Cell Counting Kit-8. The reactive oxygen species (ROS) in the cells were compared by flow cytometry. The expressions of ferroportin (FPN) and glutathione peroxidase 4 (GPX4) protein were analyzed by western blotting. The KAI1 stable expression cell line was confirmed and relabeled as MIA PaCa-2-KAI1. No significant differences in the proliferation of MIA PaCa-2 and MIA PaCa-2-KAI1 were identified. Following treatment with a ferroptosis blocker, the increase in the proliferation of MIA PaCa-2-KAI1 (from $2.06 \pm 0.02$ to $2.75 \pm 0.02$ ) was more evident compared with MIA PaCa-2 (from 2.94 \pm 0.02 to $2.95 \pm 0.02$; $\mathrm{P}<0.05)$. The ROS in MIA PaCa-2-KAI1 was significantly higher compared with MIA PaCa-2 $(\mathrm{P}<0.05)$. FPN and GPX4 protein demonstrated higher expression levels in MIA PaCa-2-KAI1 compared with MIA PaCa-2. Moreover, KAI1 exerted an obvious promotion effect on FPN expression. This study identified that the high expression of the KAI1 gene promoted the occurrence of ferroptosis in PC cells through its extensive effect on FPN and GPX4. KAI1-induced ferroptosis did not significantly inhibit the proliferation of PC cells.
\end{abstract}

\section{Introduction}

Pancreatic cancer (PC) is characterized by high mortality, low survival rate and poor prognosis. The statistics of the global

Correspondence to: Dr Xiaozhong Guo, Department of Gastroenterology, General Hospital of Northern Theater Command, 83 Wenhua Road, Shenyang, Liaoning 110840, P.R. China E-mail: guoxiaozhong1962@163.com

Key words: KAI gene, ferroptosis, pancreatic cancer cancer database (GLOBOCAN) in 2018 revealed 458,918 new patients with PC and 432,242 mortalities (1). PC incidence and mortality trends over the period of 2018-2040 are hypothesized to demonstrate an increasing trend of incidence $(+77.7 \%$ with 356,358 new cases) and mortality (+79.9\%, 345,181). Hence, PC ranks 14th in terms of cancer incidence and 7th in terms of cancer mortality (2). Despite the continuous development of medical diagnosis and treatment technology, the 5-year survival rate of PC patients remains $<10 \%$ and the median overall survival period is only 6-9 months (3). The cause of this clinical diagnosis and treatment problem is the unclear understanding of the relevant mechanism of PC cell death. Existing research results are not ideal to improve the prognosis of PC, and effective targets for inducing PC cell death remain lacking (4). Therefore, exploring the mechanism of PC cell death, the key target genes and related molecular biological mechanisms is an attractive focus for research.

In 2012, Dixon et al (5) first reported a new type of cell death mode other than apoptosis, necrosis and autophagy. This new type is characterized by the accumulation of reactive oxygen species (ROS) produced by iron metabolism and lipid peroxidation products. ROS causes oxidative damage to tissue cells and leads to cell death (6). As the process of the occurrence of ROS requires cells to be rich in available iron, the researchers termed it ferroptosis (5). This new mode of cell death has received an increasing amount of attention from researchers in recent years (7-11). Ferroptosis occurs in a variety of tumors, including $\mathrm{PC}$, and may possess an anticancer effect (4,12-14). However, the occurrence and regulatory mechanism of ferroptosis in $\mathrm{PC}$ remain to be elucidated. Identifying new ways to treat PC has been challenging. Gemcitabine, a first-line drug, is used alone or in combination for the treatment of patients with advanced pancreatic ductal adenocarcinoma (15). Heat shock 70-kDa protein 5 improves the anticancer activity of gemcitabine by inducing ferroptosis (16). As well as gemcitabine, certain traditional Chinese herbs induce ferroptosis in PC. Furthermore, a number of molecules have been demonstrated to induce ferroptosis in PC cells. These may offer new options for PC treatment (4).

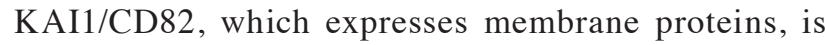
an important tumor suppressor gene. It is a prostate cancer metastasis-related suppressor gene discovered by Dong et al in 1995 (17). It belongs to the transmembrane 4 superfamily family and is located in the human chromosome 1lpl 1.2. It consists of 10 exons and 9 introns and is approximately $80 \mathrm{~kb}$ 
in length (17). The KAI1 gene is used to inhibit metastasis in PC (18-23). This gene has been recently identified to regulate the autophagy of PC cells $(24,25)$. A number of studies have provided evidence to support the association between autophagy and ferroptosis in human diseases $(26,27)$. Therefore, studying the regulatory effect of the KAI1 gene on ferroptosis in PC is warranted.

In the present study, the roles of KAI1 and ferroptosis in the onset of PC were evaluated in vitro. The objective was to provide evidence related to the KAI1 gene and its potential as a novel therapy target.

\section{Materials and methods}

Cell culture. Human PC cell lines of MIA PaCa-2 were provided by the Shanghai Institute of Cell Biology, Chinese Academy of Sciences. The cells were grown as sub-confluent monolayers in Dulbecco's modified Eagle medium (Cytiva) supplemented with $10 \%$ fetal calf serum (Cytiva), $2 \mathrm{mmol} / \mathrm{l}$ L-glutamine, $100 \mathrm{IU} / \mathrm{ml}$ penicillin $\mathrm{G}$ and $100 \mu \mathrm{g} / \mathrm{ml}$ streptomycin (Beijing Solarbio Science \& Technology Co., Ltd.) in an incubator $\left(37^{\circ} \mathrm{C}, 5 \% \mathrm{CO}_{2}\right.$ and saturated humidity). Cells were passaged at $85 \%$ confluence or were harvested for western blot analysis.

Cell transfection. KAI1 overexpression plasmid (pCMV-KAI1 DNA) was obtained from Dr Dong, Emory University School of Medicine, Atlanta, Georgia, USA (28) as a gift. According to a previous study (22), pCMV-KAI1 DNA and pCMV DNA [empty pCMV vector (Beijing Biosynthesis Biotechnology Co., Ltd.)] were transfected with a low expression cell line of KAI1: MIA PaCa-2. Cells $\left(1 \times 10^{5}\right)$ were plated in 96-well plates. When the cells reached $90-95 \%$ confluence, $1.0 \mu \mathrm{g}$ pCMV-KAI1 DNA or pCMV DNA, $1 \mu 1$ Lipofectamine ${ }^{\circledR} 2000$ reagent (Invitrogen; Thermo Fisher Scientific, Inc.), and $100 \mu \mathrm{l}$ medium without serum were mixed and incubated for $15 \mathrm{~min}$ at room temperature and then added to each well. Cells were diluted to 1,000 cells $/ \mathrm{ml}$ following transfection. The lowest concentration of $\mathrm{G} 418$ was selected in the concentration range of $100-1,000 \mu \mathrm{g} / \mathrm{ml}$. Positive clone screening was performed with $100 \mu \mathrm{g} / \mathrm{ml}$ of G418 for 4 weeks (21-23). The transfected cells were obtained through screening and passage. At $85 \%$ confluence, cells were lysed using radioimmunoprecipitation assay buffer (Beijing Solarbio Science \& Technology Co., Ltd.). The expression of the KAI1 protein was confirmed by western blot analysis.

Detection of cell viability by Cell Counting Kit-8 (CCK-8) assay. Cells in the logarithmic phase were divided into liproxstein-treated (liproxstein+) and non-liproxstein-treated (liproxstein-) groups and then inoculated into 6-well plates for $24 \mathrm{~h}$. Cells were washed twice with PBS and digested with trypsin (2.5 g/l; containing $0.2 \mathrm{~g} / 1$ EDTA). Cells were collected and centrifuged at $104.5 \mathrm{x} \mathrm{g}$ for $5 \mathrm{~min}$ at room temperature. The supernatant was discarded and the medium was gently mixed by pipetting. Cells were inoculated into 96-well plates following adjustment of the final concentration to $1 \times 10^{5}$ cells $/ \mathrm{ml}(100 \mu \mathrm{l}$ in each well). After $24 \mathrm{~h}$ of incubation, $10 \mu \mathrm{l}$ CCK-8 solution was added, and incubation was performed in an incubator for $2 \mathrm{~h}$. The absorbance was measured at a wavelength of $450 \mathrm{~nm}$ using a Multiskan FC microplate reader (Thermo Fisher Scientific, Inc.).

Detection of intracellular ROS by flow cytometric analysis. Cell suspensions from the logarithmic cells were inoculated into 6 -well plates at $2 \mathrm{ml}$ per well. When the cells completely adhered to the wall, the old culture medium was discarded. The cells were washed twice with PBS and mixed with serum-free culture medium containing H2DCFH-DA fluorescent dye (Beijing Solarbio Science \& Technology Co., Ltd.) for $30 \mathrm{~min}$ in suspension according to the manufacturer's protocol. The cells were incubated for $20 \mathrm{~min}$ in the dark at room temperature and then washed twice with precooled PBS to remove the excess dye. Cells were collected and mixed gently with $700 \mu \mathrm{l}$ PBS. The mean fluorescence intensity of intracellular ROS was detected immediately by flow cytometry (excitation wavelength: $485 \mathrm{~nm}$; emission wavelength: $530 \mathrm{~nm}$ ) using a BD FACSAria II flow cytometer (BD Biosciences). The results were analyzed with FlowJo software (version 6.1.1; FlowJo LLC).

Western blot analysis. The total proteins of logarithmic growth cells were lysed using radioimmunoprecipitation assay buffer (Beijing Solarbio Science \& Technology Co., Ltd.) and collected. Protein concentration was determined with the bicinchoninic acid method (Beijing Solarbio Science $\&$ Technology Co., Ltd.). Each sample (30 $\mu \mathrm{g} /$ lane) was resolved by $12 \%$ sodium dodecyl sulphate-polyacrylamide gel electrophoresis, followed by transfer onto a polyvinylidene difluoride membranes (EMD Millipore). The membranes were then blocked with $5 \%$ skimmed milk for $2 \mathrm{~h}$ at room temperature. Membranes were incubated with rabbit polyclonal anti-KAI1 (1:200 dilution; cat. no. sc-7179; Santa Cruz Biotechnology, Inc.), rabbit polyclonal anti-ferroportin (FPN; 1:100 dilution; cat. no. bs-3579R; BIOSS), rabbit polyclonal anti-glutathione peroxidase 4 (GPX4; 1:100 dilution; cat no bs-3884R; BIOSS) or rabbit polyclonal anti-GAPDH (1:200 dilution; cat. no. bs-0755R; BIOSS) primary antibodies overnight at $4^{\circ} \mathrm{C}$, followed by horseradish peroxidase-conjugated goat anti-rabbit IgG secondary antibody (1:2,000 dilution; cat. no. sc-2749; Santa Cruz Biotechnology, Inc.) for $1 \mathrm{~h}$ at room temperature. Immunoreactive bands were visualized using a Western Lightning Chemiluminescence Reagent Plus kit (PerkinElmer, Inc.). The signals were detected using a Las-4000 mini CCD camera (GE Healthcare). With GAPDH as the internal reference, the relative expression of protein was analyzed by densitometry using Quantity One 1-D analysis software (version 4.6.9; Bio-Rad Laboratories, Inc.) following internal reference calibration. Protein expression was derived from the grey ratio of the target band to the internal reference band.

Statistical analysis. Data were expressed as the mean \pm standard deviation of at least three independent experiments. Statistical analysis within groups was performed using repeated measures of analysis of variance followed by Bonferroni's post hoc test. Between groups analysis was performed using Student's paired t-test. SPSS 16.0 (SPSS, Inc.) was used for all statistical analyses. $\mathrm{P}<0.05$ was considered to indicate a statistically significant difference. 

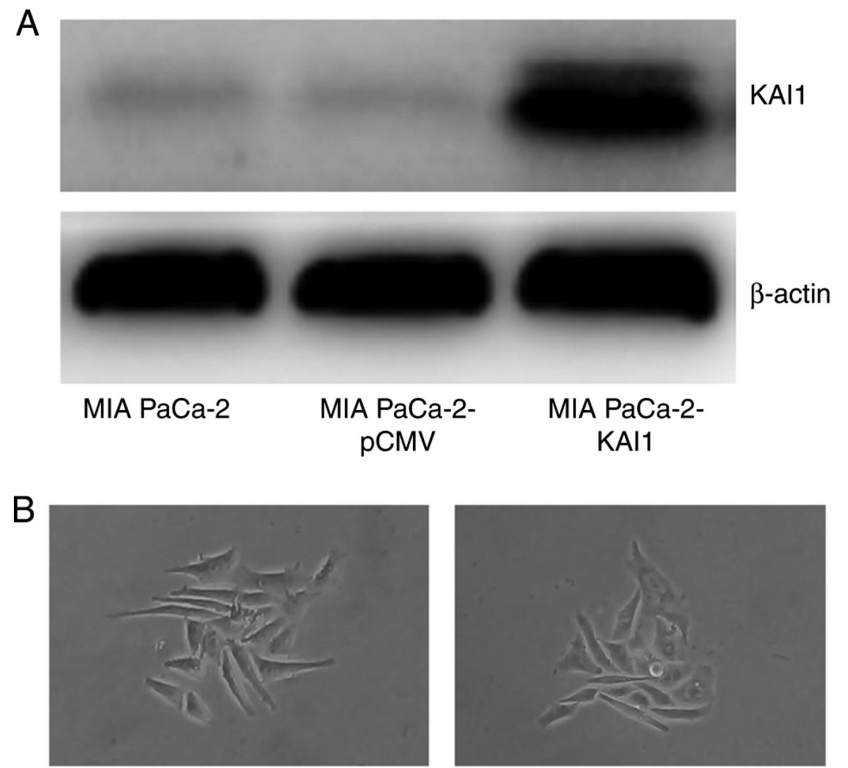

MIA PaCa-2

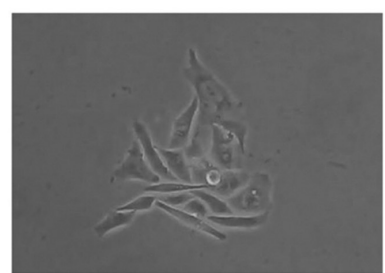

MIA PaCa-2-KAl1

Figure 1. Transfection efficiency of pCMV-KAI1. (A) Western blot analysis results for untransfected MIA PaCa-2 cells and MIA PaCa-2 cells transfected with pCMV-KAI1 DNA and pCMV DNA. MIA PaCa-2 cells transfected with pCMV-KAI1 DNA demonstrated high KAI1 expression levels, whereas the untransfected cells and transfected with empty pCMV DNA cells demonstrated low KAIl expression levels. (B) Phase-contrast photomicrographs of morphology of MIA PaCa-2 cells before and after transfection with pCMV-KAI1 DNA (original magnification, x200).

\section{Results}

Construction of KAII stable expression. Western blot analysis revealed that the MIA PaCa-2 line transfected with KAI1 plasmid exhibited strong protein bands, whereas the untransfected cells and transfected with empty pCMV vector cells exhibited weak bands (Fig. 1A). MIA PaCa-2-KAI1 cell morphology did not show the scattering and acquisition of fusiform and fibroblastic phenotype compared with MIA PaCa-2 cells (Fig. 1B). The MIA PaCa-2 cells that demonstrated a stable expression of the KAI1 gene were labelled as MIA PaCa-2-KAI1.

Cell viability. CCK-8 detection revealed that KAI1 overexpression had no significant effect on the viability of MIA PaCa-2. Following treatment with liproxstein, a type of ferroptosis inhibitor, for $24 \mathrm{~h}$, the increased viability of MIA PaCa-2-KAI1 (from $2.06 \pm 0.02$ to $2.75 \pm 0.02$ ) was more apparent compared with that of MIA PaCa-2 (from 2.94 \pm 0.02 to $2.95 \pm 0.02 ; \mathrm{P}<0.05$; Fig. 2), thereby indicating that KAI1 may promote ferroptosis.

ROS are affected by KAII. As demonstrated by flow cytometry results (Fig. 3), the fluorescence intensity of ROS in MIA PaCa-2-KAI1 (43.63\%) was significantly higher compared with that in MIA PaCa-2 (1.42\%; $\mathrm{P}<0.05)$. This suggested that KAI1 overexpression can induce ROS production in MIA $\mathrm{PaCa}-2$ cells.

KAIl gene promotes FPN and GPX4 protein expressions. Western blot analysis was used to detect the proteins in the MIA

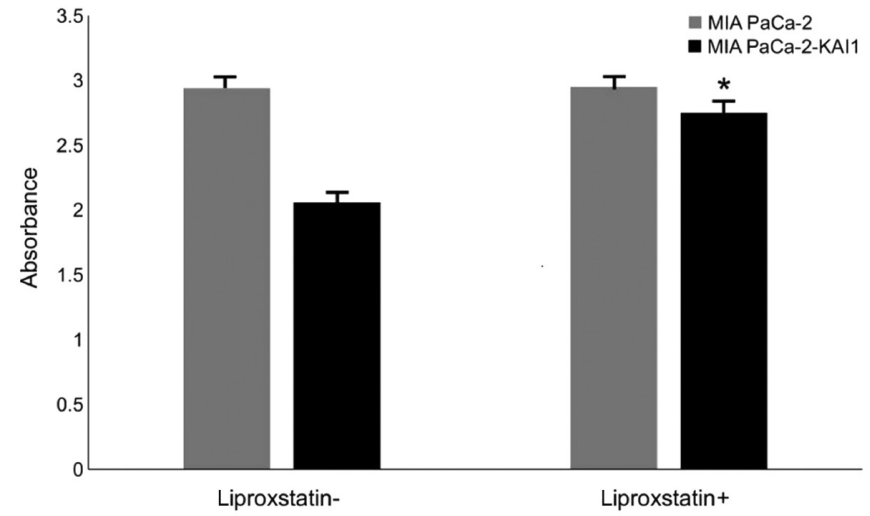

Figure 2. Cell viability curve of MIA PaCa-2-KAI1 and MIA PaCa-2 cells according to the CCK-8 assay findings. With liproxstein, the increased viability of MIA PaCa-2-KAI1 was more marked compared with that of MIA $\mathrm{PaCa}-2\left({ }^{*} \mathrm{P}<0.05\right)$.

PaCa-2 and MIA PaCa-2-KAI1 cells. FPN and GPX4 protein expressions were higher in MIA PaCa-2-KAI1 compared with MIA PaCa-2 (Fig. 4A). The comparison of the grey values, with GAPDH protein as the internal control, indicated that KAI1 promoted FPN protein expression (Fig. 4B).

\section{Discussion}

Previous studies have shown that ferroptosis inhibits the growth and proliferation of PC cells, thereby providing a new approach for the treatment of PC (18-25). In the present study, the widely-studied KAI1 gene was the research object. The KAI1 gene was highly expressed in the MIA PaCa- 2 cells by transfection and the occurrence of ferroptosis was detected. In the CCK-8 experiment, it was identified that the viability of MIA PaCa- 2 cells was insignificantly inhibited by KAI1 overexpression and that the inhibition was weakened by further treatment with the ferroptosis inhibitor liproxstein. It was hypothesized that the inhibitor blocked KAI1-induced ferroptosis. KAI1 induced the MIA PaCa-2 cells to produce ROS extensively, as detected by flow cytometry. Previous studies have confirmed that ROS serve an important role in promoting ferroptosis (29-32). Previous research in our laboratory demonstrated that KAI1 induced autophagy in MIA PaCa-2 cells $(24,25)$. Previous studies have shown that the activation of the autophagy pathway promotes ferroptosis in fibroblasts and cancer cells $(26,27)$. On the basis of the experimental results from the present study and literature reports, it was hypothesized that KAIl overexpression can promote the ferroptosis of PC cells.

To identify the possible mechanism underlying this promoting effect, the changes in cell protein expression was detected. Genes, such as FPN, GPX4, system Xc-, transferrin, transferrin receptor 1, divalent metal transporter 1, ferritin heavy chain 1, and ferritin lightchain, are the critical mediators in the ferroptosis procedure (33). FPN and GPX4 were markedly expressed from the MIA PaCa-2 cells, which highly express KAI1. In addition, the expression of FPN was greatly increased according to the densitometry analysis. Previous studies have confirmed that FPN is a multidomain 

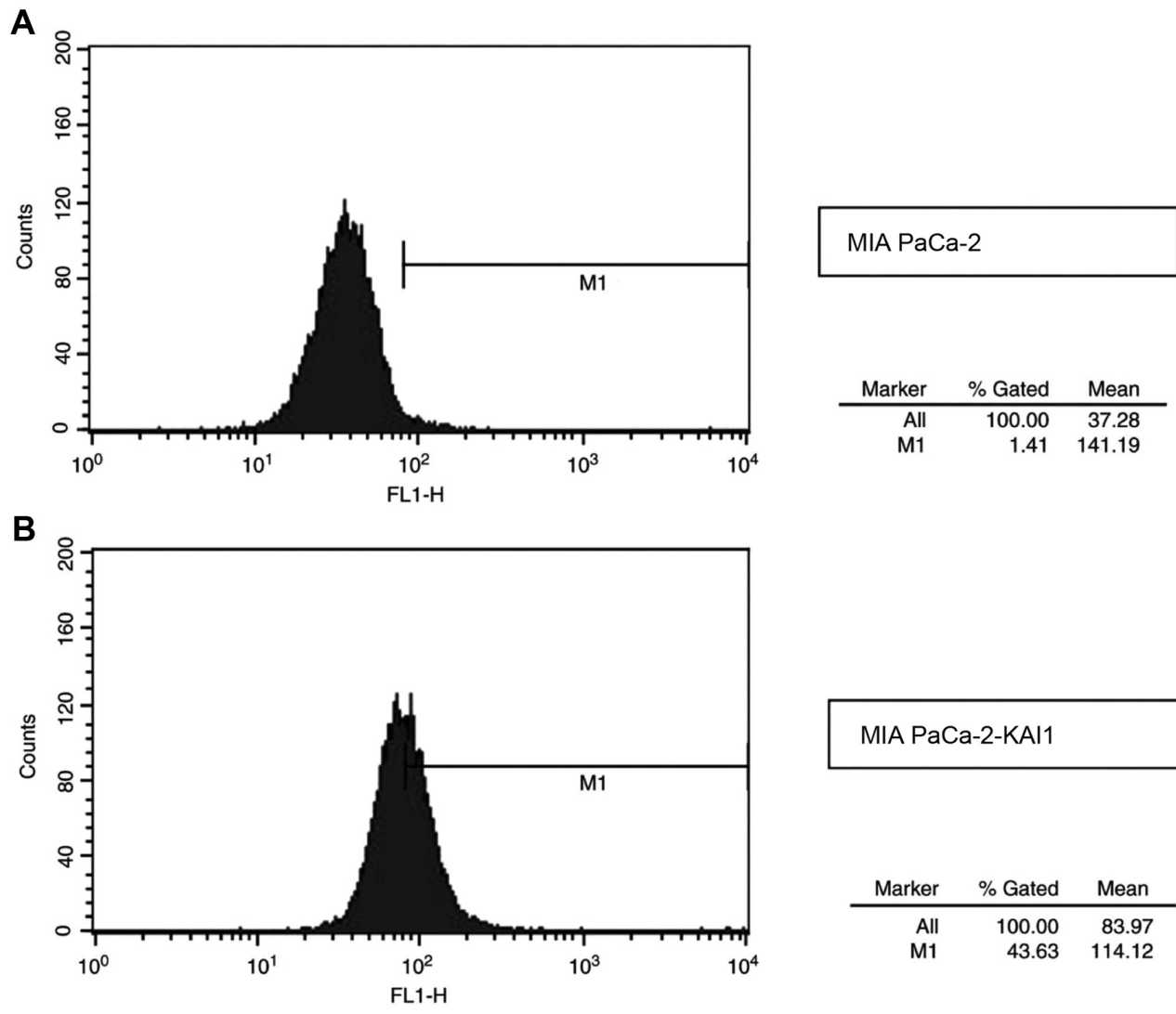

MIA PaCa-2-KAI1

C

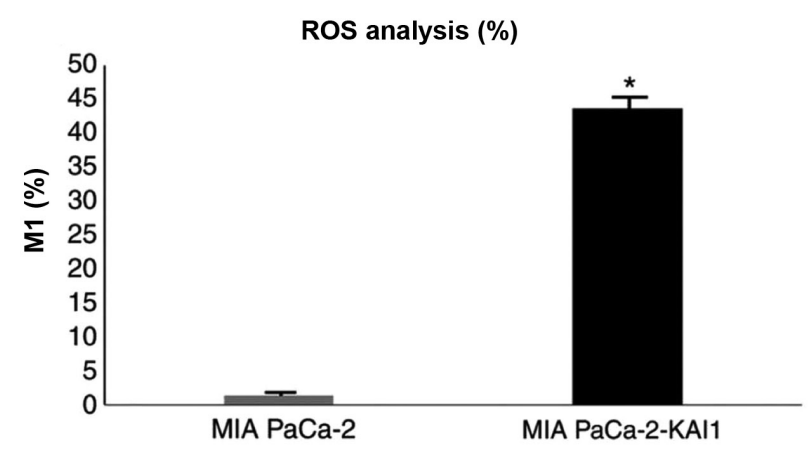

Figure 3. Flow cytometry results demonstrate that the KAI1 gene can promote ROS expression. In the histogram, the expression of ROS in the cells is depicted by the black-colored area. (A) In MIA PaCa-2 cells, $\sim 1.41 \%$ positive cells were identified. (B) In MIA PaCa-2-KAI1 cells, 46.63\% positive cells were identified. (C) The detection intensity of ROS in MIA PaCa-2-KAI1 cells was significantly higher compared with MIA PaCa-2 cells ("P<0.05). ROS, reactive oxygen species.

A

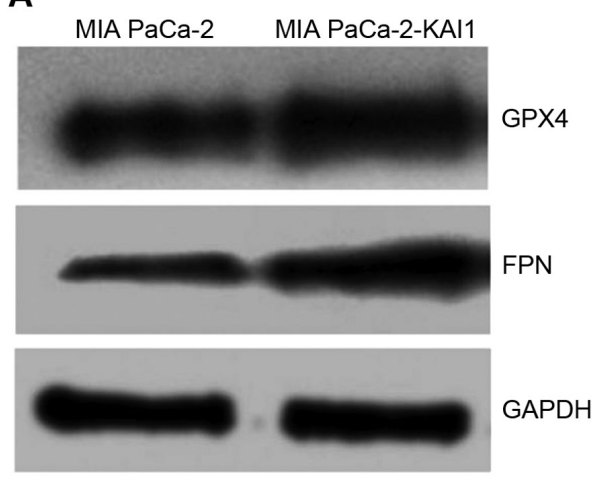

B

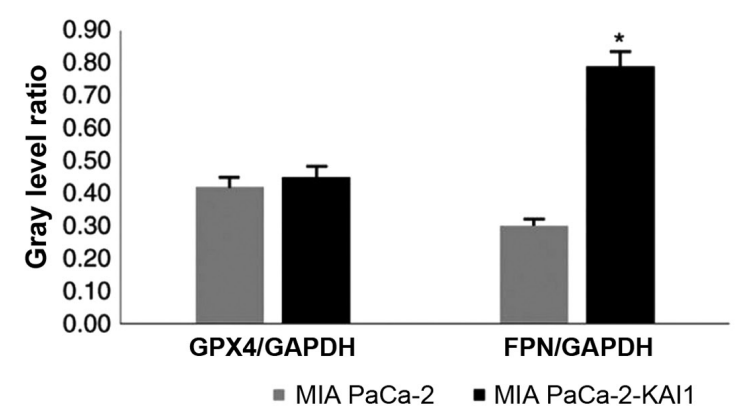

Figure 4. FPN and GPX4 protein expressions detected by western blot analysis. (A) FPN and GPX4 protein expressions were higher in the MIA PaCa-2-KAI1 cells compared with the MIA PaCa-2 cells. (B) The FPN grey value, with GAPDH protein as the internal control, was increased more notably in the MIA PaCa-2-KAI1 cells compared with the MIA PaCa-2 cells ("P<0.05). No significant difference was observed in the GPX4 grey value between the MIA PaCa-2-KAI1 and MIA PaCa-2 cells. FPN, ferroportin; GPX4, glutathione peroxidase 4. 
transmembrane protein encoded by SLC40A and is the only existing transmembrane iron export protein $(6,34)$. Studies on a variety of tumor cells have shown that the high expression of FPN can cause iron outflow, which can lead to iron deficiency in cells and inhibit cancer cell growth (35-38). Therefore, the results from the present study, indicating the promotion of FPN expression by KAI1, further confirmed that KAI1 overexpression can inhibit the proliferation of PC cells. In addition, increasing iron intake and reducing iron storage may lead to iron overload, which may in turn lead to ferroptosis $(39,40)$. Therefore, it was hypothesized that the high expression of KAI1 promoted FPN expression and led to the transportation of excessive $\mathrm{Fe}^{2+}$ out of the cells, thereby causing the cancer cells to absorb large amounts of $\mathrm{Fe}^{3+}$. This condition breaks the iron homeostasis in cancer cells. Excessive iron transforms $\mathrm{H}_{2} \mathrm{O}_{2}$ and lipoperoxidation into ROS through the Fenton reaction and finally leads to ferroptosis (6).

The loss of GPX4 activity is another major source of ROS and is a key factor for inducing ferroptosis (41-43). The results of the present study demonstrated that KAI1 can promote the expression of GPX4, although it may inhibit the occurrence of ferroptosis. Nevertheless, the effect of KAI1 on GPX4 expression was weaker compared with that on FPN expression. Therefore, the high expression of the KAI1 gene promoted the occurrence of ferroptosis in PC cells through its comprehensive effects on FPN and GPX4. The limitation of the present study that should be acknowledged when interpreting the results is the lack of research on the in-depth mechanism underlying the effects of KAI1 on FPN and GPX4, as well as the relationship between KAI1 and other ferroptosis-related mediators.

As indicated by the results of the proliferation experiments, KAI1-induced ferroptosis did not significantly inhibit the proliferation of PC cells. According to our previous findings on the KAI1 gene, it inhibits the epithelial-mesenchymal transition and the migration and lymphangiogenesis of PC cells (23). It was concluded that although the inhibition of proliferation was not significant, it still limited the metastasis of PC cells to some extent. The present study added to the theoretical basis of KAI1 as a tumor suppressor gene and provided a new experimental basis for its clinical application.

\section{Acknowledgements}

The authors would like to thank Dr Dong Jintang (Emory University School of Medicine, Atlanta, USA) for providing the KAI1 overexpression plasmid (pCMV-KAI1 DNA).

\section{Funding}

The present study was supported by grants from the Science Foundation of Liaoning (grant no. 2019-MS-352) and the Science and Technology Foundation of Shenyang (grant no. 19-112-4-046).

\section{Availability of data and materials}

The datasets used and/or analyzed during the current study are available from the corresponding author on reasonable request.

\section{Authors' contributions}

XL and XG designed the study. XL and JC performed the experiments and wrote the first draft. XL, HL and $\mathrm{HH}$ collected and analyzed the data. All authors contributed to the design and interpretation of the study and to further drafts of the manuscript. All authors reviewed and approved the final manuscript.

\section{Ethics approval and consent to participate}

Not applicable.

\section{Patient consent for publication}

Not applicable.

\section{Competing interests}

The authors declare that they have no competing interests.

\section{References}

1. Bray F, Ferlay J, Soerjomataram I, Siegel RL, Torre LA and Jemal A: Global cancer statistics 2018: GLOBOCAN estimates of incidence and mortality worldwide for 36 cancers in 185 countries. CA Cancer J Clin 68: 394-424, 2018.

2. Siegel RL, Miller KD and Jemal A: Cancer statistics, 2018. CA Cancer J Clin 68: 7-30, 2018.

3. Rawla P, Sunkara T and Gaduputi V: Epidemiology of pancreatic cancer: Global trends, etiology and risk factors. World J Oncol 10: 10-27, 2019.

4. Chen G, Guo GQ, Zhou XD and Chen HX: Potential mechanism of ferroptosis in pancreatic cancer. Oncol Lett 19: 579-587, 2020.

5. Dixon SJ, Lemberg KM, Lamprecht MR, Skouta R, Zaitsev EM, Gleason CE, Patel DN, Bauer AJ, Cantley AM, Yang WS, et al: Ferroptosis: An iron-dependent form of nonapoptotic cell death. Cell 5: 1060-1072, 2012.

6. Kajarabille N and Latunde-Dada GO: Programmed cell-death by ferroptosis: Antioxidants as mitigators. Int J Mol Sci 19: 4968, 2019.

7. Hasegawa M, Takahashi H, Rajabi H, Alam M, Suzuki Y Yin L, Tagde A, Maeda T, Hiraki M, Sukhatme VP and Kufe D: Functional interactions of the cystine/glutamate antiporter, CD44v and MUC1-C oncoprotein in triple-negative breast cancer cells. Oncotarget 7: 11756-11769, 2016.

8. Lin R, Zhang Z, Chen L, Zhou Y, Zou P, Feng C, Wang L and Liang G: Dihydroartemisinin (DHA) induces ferroptosis and causes cell cycle arrest in head and neck carcinoma cells. Cancer Lett 381: 165-175, 2016.

9. Tang M, Chen Z, Wu D and Chen L: Ferritinophagy/ferroptosis: Iron-related newcomers in human diseases. J Cell Physiol 233: 9179-9190, 2018.

10. Nie J, Lin B, Zhou M, Wu L and Zheng T: Role of ferroptosis in hepatocellular carcinoma. J Cancer Res Clin Oncol 144: 2329-2337, 2018.

11. Xia XJ, Fan XP, Zhao MY and Zhu P: The relationship between ferroptosis and tumors-a novel landscape for therapeutic approach. Curr Gene Ther 19: 117-124, 2019.

12. Yamaguchi Y, Kasukabe T and Kumakura S: Piperlongumine rapidly induces the death of human pancreatic cancer cells mainly through the induction of ferroptosis. Int J Oncol 3: 1011-1022, 2018.

13. Wang K, Zhang Z, Wang M, Cao X, Qi J, Wang D, Gong A and Zhu H: Role of GRP78 inhibiting artesunate-induced ferroptosis in KRAS mutant pancreatic cancer cells. Drug Des Devel Ther 13: 2135-2144, 2019.

14. Song Z, Xiang X, Li J, Deng J, Fang Z, Zhang L and Xiong J: Ruscogenin induces ferroptosis in pancreatic cancer cells. Oncol Rep 43: 516-524, 2020.

15. Binenbaum Y, Na'ara S and Gil Z: Gemcitabine resistance in pancreatic ductal adenocarcinoma. Drug Resist Updat 23: 55-68, 2015. 
16. Zhu S, Zhang Q, Sun X, Zeh HJ III, Lotze MT, Kang R and Tang D: HSPA5 regulates ferroptotic cell death in cancer cells. Cancer Res 77: 2064-2077, 2017.

17. Dong JT, Lamb PW, Rinker-Schaeffer CW, Vukanovic J, Ichikawa T, Isaacs JT and Barrett JC: KAI1, a metastasis suppressor gene for prostate cancer on human chromosome 11p11.2. Science 268: 884-886, 1995.

18. Guo XZ, Friess H, Shao XD, Liu MP, Xia YT, Xu JH and Buchler MW: KAI1 gene is differently expressed in papillary and pancreatic cancer influence on metastasis. World J Gastroenterol 6: 866-871, 2000.

19. Xu JH, Guo XZ, Ren LN, Shao LC and Liu MP: KAI1 is potential target on anti-metastasis in pancreatic cancer cells. World J Gastroenterol 14: 1126-1132, 2008.

20. Liu X, Guo XZ, Zhang WW, Lu ZZ, Zhang QW, Duan HF and Wang LS: KAI1 inhibits HGF-induced invasion of pancreatic cancer by sphingosine kinase activity. Hepatobiliary Pancreat Dis Int 10: 201-208, 2011.

21. Liu X, Guo XZ, Li HY, Chen J, Ren LN and Wu CY: KAI1 inhibits lymphangiogenesis and lymphatic metastasis of pancreatic cancer in vivo. Hepatobiliary Pancreat Dis Int 13: 87-92, 2014.

22. Liu X, Guo X, Li H, Chen J and Qi X: Src/STAT3 signaling pathways are involved in KAI1-induced downregulation of VEGF-C expression in pancreatic cancer. Mol Med Report 13: 4774-4778, 2016.

23. Liu X, Guo XZ, Li HY and Chen J: KAI1 reverses the epithelial-mesenchymal transition in human pancreatic cancer cells. Hepatobiliary Pancreat Dis Int 18: 471-477, 2019.

24. Wu CY, Yan J, Yang YF, Xiao FJ, Li QF, Zhang QW, Wang LS, Guo XZ and Wang H: Overexpression of KAIl induces autophagy and increases MiaPaCa-2 cell survival through the phosphorylation of extracellular signal-regulated kinases. Biochem Biophys Res Commun 404: 802-808, 2011.

25. Wu CY, Guo XZ and Li HY: Hypoxia and serum deprivation protected MiaPaCa-2 cells from KAI1-induced proliferation inhibition through autophagy pathway activation in solid tumors. Clin Transl Oncol 17: 201-208, 2015.

26. Hou W, Xie Y, Song X, Sun X, Lotze MT, Zeh HJ III, Kang R and Tang D: Autophagy promotes ferroptosis by degradation of ferritin. Autophagy 12: 1425-1428, 2016.

27. Zhou B, Liu J, Kang R, Klionsky DJ, Kroemer G and Tang D: Ferroptosis is a type of autophagy-dependent cell death. Semin Cancer Biol 66: 89-100, 2020.
28. Gao AC, Lou W, Dong JT, Barrett JC, Danielpour D and Isaacs JT: Defining regulatory elements in the human KAI1 (CD 82) metastasis suppressor gene. Prostate 57: 256-260, 2003.

29. Xie Y, Hou W, Song X, Yu Y, Huang J, Sun X, Kang R and Tang D: Ferroptosis: Process and function. Cell Death Differ 23: 369-379, 2016

30. Lu B, Chen XB, Ying MD, He QJ, Cao J and Yang B: The role of ferroptosis in cancer development and treatment response. Front Pharmacol 8: 992, 2018.

31. Dixon SJ and Stockwell BR: The role of iron and reactive oxygen species in cell death. Nat Chem Biol 10: 9-17, 2014.

32. Li J, Cao F, Yin HL, Huang ZJ, Lin ZT, Mao N, Sun B and Wang G: Ferroptosis: Past, present and future. Cell Death Dis 11: $88,2020$.

33. Mou Y, Wang J, Wu J, He D, Zhang C, Duan C and Li B: Ferroptosis, a new form of cell death: Opportunities and challenges in cancer. J Hematol Oncol 12: 34-50, 2019.

34. Camaschella C, Nai A and Silvestri L: Iron metabolism and iron disorders revisited in the hepcidin era. Haematologica 105: 260-272, 2020

35. Manz DH, Blanchette NL, Paul BT, Torti FM and Torti SV: Iron and cancer: Recent insights. Ann N Y Acad Sci 1368: 149-161, 2016.

36. Petronek MS, Spitz DR, Buettner GR and Allen BG: Linking cancer metabolic dysfunction and genetic instability through the lens of iron metabolism. Cancers (Basel) 11: 1077, 2019.

37. Kazan HH, Urfali-Mamatoglu C and Gunduz U: Iron metabolism and drug resistance in cancer. Biometals 30: 629-641, 2017.

38. Thévenod F: Iron and its role in cancer defense: A double-edged sword. Met Ions Life Sci 18, 2018.

39. Chen Y, Fan Z, Yang Y and Gu C: Iron metabolism and its contribution to cancer (Review). Int J Oncol 54: 1143-1154, 2019.

40. Wang YF, Yu L, Ding J and Chen Y: Iron metabolism in cancer. Int J Mol Sci 20: 95, 2019.

41. Forcina GC and Dixon SJ: GPX4 at the crossroads of lipid homeostasis and ferroptosis. Proteomics 19: e1800311, 2019.

42. Seibt TM, Proneth B and Conrad M: Role of GPX4 in ferroptosis and its pharmacological implication. Free Radic Biol Med 133: 144-152, 2019

43. Yang WS, SriRamaratnam R, Welsch ME, Shimada K, Skouta R, Viswanathan VS, Cheah JH, Clemons PA, Shamji AF, Clish CB, et al: Regulation of ferroptotic cancer cell death by GPX4. Cell 156: 317-331, 2014. 